\title{
Evaluation of the Effects of Different Bacteroides vulgatus Strains against DSS-Induced Colitis
}

\author{
Sijia Li, ${ }^{1,2}$ Chen Wang,,2 Chengcheng Zhang, ${ }^{1,2}$ Yanhong Luo,,2 Qianqian Cheng, \\ Leilei Yu, ${ }^{1,2}$ and Zhen Sun $\left(\mathbb{D}^{1}\right.$ \\ ${ }^{1}$ School of Food Science and Technology, Jiangnan University, Wuxi, Jiangsu 214122, China \\ ${ }^{2}$ State Key Laboratory of Food Science and Technology, Jiangnan University, Wuxi, Jiangsu 214122, China \\ Correspondence should be addressed to Zhen Sun; zhsun@jiangnan.edu.cn
}

Received 17 April 2021; Revised 10 May 2021; Accepted 18 May 2021; Published 30 May 2021

Academic Editor: Tingtao Chen

Copyright (C) 2021 Sijia Li et al. This is an open access article distributed under the Creative Commons Attribution License, which permits unrestricted use, distribution, and reproduction in any medium, provided the original work is properly cited.

\begin{abstract}
Although the strain-dependent effects of Bacteroides vulgatus on alleviating intestinal inflammatory diseases have been demonstrated, the literature has rarely focused on the underlying causes of this effect. In this study, we selected four $B$. vulgatus strains (FTJS5K1, FTJS7K1, FSDTA11B14, and FSDLZ51K1) with different genomic characteristics and evaluated their protective roles against dextran sulfate sodium- (DSS-) induced colitis. Compared to the other three tested strains, B. vulgatus $7 \mathrm{~K} 1$ more strongly ameliorated the DSS-induced weight loss, shortening of the colon length, increased disease activity index scores, colonic tissue injury, and immunomodulatory disorder. In contrast, B. vulgatus 51K1 significantly worsened the DSS-induced alterations in the tumor necrosis factor-alpha (TNF- $\alpha$ ) concentration and colonic histopathology. A comparative genomic analysis of $B$. vulgatus $7 \mathrm{~K} 1$ and $51 \mathrm{~K} 1$ showed that the beneficial effects of $B$. vulgatus $7 \mathrm{~K} 1$ may be associated with some of its specific genes involved in the production of short-chain fatty acids or capsular polysaccharides and enhancement of its survivability in the gut. In conclusion, these findings indicate that the supplementation of $B$. vulgatus $7 \mathrm{~K} 1$ is a potentially efficacious intervention for alleviating colitis and provides scientific support for the screening of probiotics with anticolitis effect.
\end{abstract}

\section{Introduction}

Bacteroides, one of the most abundant genera in the mammalian colon, has been a primary candidate for next-generation probiotics and has attracted considerable attention due to its role in the prevention of a series of metabolic disorders, including obesity $[1,2]$, diarrhea [3], viral encephalitis [4], and enteritis [5]. In particular, the protective effects of Bacteroides on inflammatory diseases in the gut are a hot topic. The results of human studies have indicated that the relative abundance of Bacteroides in patients with inflammatory bowel disease (IBD) is markedly lower than that in healthy participants $[6,7]$. Furthermore, animal studies related to colitis have demonstrated that several strains of Bacteroides, including Bacteroides fragilis NCTC 9343, Bacteroides thetaiotaomicron DSM 2079, and Bacteroides cellulosilyticus DSM 14838, can expand the population of interleukin- (IL-) 10 -producing $\mathrm{CD}^{+}{ }^{+} \mathrm{CD} 45 \mathrm{RB}^{\text {low }} \mathrm{T}$ cells
[8], ameliorate the histopathological damage of the gut [9], and increase the levels of anti-inflammatory IL-10 and Treg cells [10]. These findings indicate that Bacteroides strains could be beneficial to the restoration of gut health in patients with intestinal inflammatory diseases. However, some studies have shown inconsistent results. One study found that the oral administration of enterotoxigenic B. fragilis 86-5443-2-2 isolated from piglets induced colitis in mice, which was manifested by the severe damage of colon tissue [11]. In addition, Bacteroides eggerthii 12986 has been reported to reduce survival, accelerate body weight loss, and increase intestinal bleeding in dextran sulfate sodium- (DSS-) treated mice, which then enhanced the severity of their colitis [12]. These reports suggest that the effects of Bacteroides on intestinal inflammatory diseases are species or even strain-specific.

The varying effects of different strains may be attributable to their physiological characteristics. The colonization ability of strains is a physiological characteristic related to the 
protective functions of some strains against intestinal inflammatory disease. The type VI secretion system (T6SS) [13], antibacterial proteins [14], or capsular polysaccharides [15] of certain Bacteroides strains may increase their competitive fitness in the gut. Colitis-related studies have indicated that a high competitive colonization ability of $B$. fragilis could inhibit the intestinal adhesion of and further exposure to toxic pathogenic bacteria, and thus, prevent colitis [16]. The compounds produced by various strains also play a role in the development of intestinal inflammatory disease. For example, short-chain fatty acids (SCFAs), especially butyrate, may promote the intestinal epithelial barrier function [17], inhibit the central regulator of the inflammatory NF- $\kappa \mathrm{B}$ signaling pathway [18], and decrease oxidative stress [19], thereby preventing pathological damage of the colon associated with intestinal inflammatory disease. One study found that the administration of $B$. fragilis could improve the tight junction (TJ) integrity of the gut by increasing the number of SCFAs [20]. However, enterotoxins secreted by B. fragilis VPI 13784 have been reported to induce inflammation and significant colon tissue damage in lambs, rabbits, and rats after injection of the strain into their intestinal ligated loops [21]. The surface antigens of some strains can also affect intestinal inflammatory diseases. For example, polysaccharide $\mathrm{A}$, a type of capsular polysaccharide present on $B$. fragilis NCTC 9343, has been reported to alleviate colitis [22] and colitis-associated colorectal cancer [23]. Another capsular zwitterionic polysaccharide TP2 from $B$. fragilis ZY-312 has been reported to protect rats from 2,4-dinitrobenzenesulfonic acid-triggered enteritis by reducing the degree of intestinal adhesion and the area of intestinal ulcers [5]. Moreover, the sphingolipids of $B$. fragilis NCTC9343 have been found to attenuate oxazolone-induced experimental colitis [24]. These results indicate that the effects of Bacteroides and even probiotics on alleviating intestinal inflammatory diseases are closely related to their physiological characteristics. Notably, the phenotype of bacteria is determined by their genome, and some studies have revealed that the functional differences between various probiotic strains on colitis remission are strongly associated with their genome $[25,26]$.

Bacteroides vulgatus is a representative species of the Bacteroides genus and is known to have a beneficial effect on the human colon health $[27,28]$. Some studies have reported that $B$. vulgatus mpk can inhibit Escherichia coliinduced colitis [29] or Yersinia enterocolitica-induced inflammation [30], whereas other studies have demonstrated the proinflammatory effect of certain B. vulgatus strains. One study showed that $B$. vulgatus DESEP-B could induce colitis in HLA-B27 transgenic rats [31]. Another study found that B. vulgatus TUSVM 40G2-33 led to the enhancement of carrageenan-induced colitis in guinea pigs [32]. These results imply that the protective effect of $B$. vulgatus is straindependent. Furthermore, a previous study has revealed the considerable differences in inflammatory responses of guinea pigs administrated with seven different $B$. vulgatus strains in an experimental model for ulcerative colitis [33]. This demonstrated the variable ability of various $B$. vulgatus strains in the enhancement of colitis. However, most investigations of the effect of B. vulgatus on colitis have only focused on a single strain. Hence, it is important to investigate the complex relationship between $B$. vulgatus strains and colitis and the reasons for the varying effects of different strains. In this study, we selected four $B$. vulgatus strains with large differences in their genomes and assessed their roles in alleviating colitis. Due to its simplicity, reliability, and applicability, we used DSS to induce colitis in mice [34]. We then analyzed the genomic characteristics of the selected $B$. vulgatus strains to identify the functional genes that may play a role in alleviating the intestinal damage caused by the DSS.

\section{Materials and Methods}

2.1. Bacterial Strains and Preparation. We used B. vulgatus strains FTJS5K1 (5K1), FTJS7K1 (7K1), FSDTA11B14 (11B14), and FSDLZ51K1 (51K1) in this study, all of which had been isolated from the fecal samples of different volunteers. The $5 \mathrm{~K} 1$ and $7 \mathrm{~K} 1$ strains were deposited in the Culture Collection of Food Microorganisms (CCFM) of Jiangnan University (Wuxi, China). The four strains were grown anaerobically at $37^{\circ} \mathrm{C}$ for $18 \mathrm{~h}$ in a brain-heart infusion broth (Hopebio, China) supplemented with $5 \mu \mathrm{g} / \mathrm{mL}$ hemin (Sangon Biotech, China) and $0.5 \mu \mathrm{g} / \mathrm{mL}$ vitamin K1 (Sangon Biotech, China). A fresh culture of each strain was collected by centrifugation ( $5 \mathrm{~min}$ at $6000 \mathrm{~g}$ ) and then washed twice with sterile phosphate-buffered saline (PBS). The final bacterial pellets were resuspended in sterile $\mathrm{PBS}$ at a concentration of $5 \times 10^{9}$ colony-forming units (CFUs) $/ \mathrm{mL}$.

2.2. Animal Experimental Design. Sixty specific pathogenfree C57BL/6J mice (male, 6 weeks old) purchased from the Shanghai Laboratory Animal Center were housed at five animals per cage at the Animal Experiment Center of Jiangnan University. The mice were provided with sufficient sterilized water and standard food ad libitum and were maintained under standard conditions $\left(20-24^{\circ} \mathrm{C}, 50 \%-60 \%\right.$ humidity, and a $12 \mathrm{~h}$ light/darkness cycle). All mice were given a 7 day period to acclimatize to their new environment. Then, they were randomly divided into six groups (10 mice in each group): control, DSS, DSS $+5 \mathrm{~K} 1, \mathrm{DSS}+7 \mathrm{~K} 1, \mathrm{DSS}+51 \mathrm{~K} 1$, and DSS+11B14. To induce acute colitis in the mice in the experimental groups, 3\% DSS (36-50 kDa, MP Biomedicals, Carlsbad, CA, USA) was added to their sterile filtered drinking water for 7 days. During the trial, the mice in the control and DSS groups were orally gavaged with $0.2 \mathrm{~mL}$ of sterile PBS. The mice in the other groups were fed one of the four B. vulgatus strains at a dose of $1 \times 10^{9} \mathrm{CFUs} / 0.2 \mathrm{~mL}$ in sterile PBS by gavage. Three essential parameters of the disease activity index (DAI) were measured daily [35], including body weight, stool consistency, and fecal blood. Fecal occult blood was measured by using an Occult Blood Kit (Nanjing Jiancheng Co., Ltd., Nanjing, China). On day 7 after treatment, fresh stool samples were collected and instantly frozen at $-80^{\circ} \mathrm{C}$ for further analysis. On day 8 , euthanasia of mice was performed by carbon dioxide administration. The colons of all the mice were extracted, and their lengths were measured. The distal colon $(5 \mathrm{~mm})$ was then immersed in a $4 \%$ paraformaldehyde solution, and the remainder was stored at $-80^{\circ} \mathrm{C}$ for subsequent testing. All the procedures related 
TABle 1: Primers sequences used for RT-qPCR.

\begin{tabular}{lcc}
\hline Gene & Forward & Reverse \\
\hline Claudin-1 & $5^{\prime}$-GATGTGGATGGCTGTCATTG-3' & $5^{\prime}$-CCTGGCCAAATTCATACCTG-3' \\
Occludin & $5^{\prime}$-CACACTTGCTTGGGACAGAG-3' & $5^{\prime}$-TAGCCATAGCCTCCATAGCC-3' \\
ZO-1 & $5^{\prime}$-CTTCTCTTGCTGGCCCTAAAC-3' & $5^{\prime}$-TGGCTTCACTTGAGGTTTCTG-3' \\
Mucin 2 & $5^{\prime}$-TGCCCACCTCCTCAAAGAC-3' & $5^{\prime}$-GTAGTTTCCGTTGGAACAGTGAA-3' \\
$\beta$-Actin & $5^{\prime}$-GGCTGTATTCCCCTCCATCG-3' & $5^{\prime}$-CCAGTTGGTAACAATGCCATGT-3' \\
\hline
\end{tabular}

to these animal experiments were approved by the Committee of Ethics in Jiangnan University, China (JN. NO. 20190930c0801120[249]).

\subsection{Genome Sequencing, Clusters of Orthologous Group} (COG) Annotation, and Phylogenetic Tree Construction. Genome sequencing of B. vulgatus strains was performed using the Illumina HiSeq System by Majorbio (China), as described in a previous study [36, 37]. GLIMMER software was used to predict the protein-coding sequences. To identify the relationships between different $B$. vulgatus strains, we used OrthoMCL1.4 to generate the orthologous genes of 14 strains. Among these 14 strains, the genome of ATCC 8482 (Genome accession number: NC_009614.1), mpK (Genome accession number: CP013020.1), PC510 (Genome accession number: NZ_ADKO00000000.1), AF34-15 (Genome accession number: NZ_QRPW00000000.1), AM44-21 (Genome accession number: NZ_QSEZ00000000.1), RH 1270 (Genome accession number: NZ_WCIG00000000.1), and RJ2H1 (Genome accession number: NZ_PHJG00000000.1) was downloaded from the National Center for Biotechnology Information (NCBI) database. The other 7 genome sequences of 5K1 (Genome accession number: JACBPX0000000000), 7K1 (Genome accession number: JACBPY000000000), 11B14 (Genome accession number: JACBPW000000000), 51K1 (Genome accession number: JACBPV000000000), FBJS10K3 (Genome accession number: JACBPS000000000), FGSZY37K4 (Genome accession number: JACBPT000000000), and FJSWX62K35 (Genome accession number: JACBPU000000000) were from the current study. We then used PhyML3 software to construct a neighbor-joining phylogenetic tree based on the core gene alignment generated using MAFFT [38]. To distinguish the functional genes between the various strains, we annotated the gene functions against the Carbohydrate-Active enZyme (CAZy) database and the Clusters of Orthologous Groups (COG) protein database by BLASTp [39].

2.4. Determination of Intestinal Permeability. To assess the intestinal permeability of the mice, we used 4000-Da fluorescein isothiocyanate-dextran (DX-4000-FITC, Sigma-Aldrich, USA), as described in [40]. Briefly, the mice were orally administrated DX-4000-FITC at a dosage of $500 \mathrm{mg} / \mathrm{kg}$ of body weight after fasting for $6 \mathrm{~h}$ on day 7 . After $1 \mathrm{~h}$, their blood was collected to detect the concentration of DX4000-FITC.
2.5. Histological Evaluation. The fixed colon tissues were embedded in paraffin, stained with hematoxylin and eosin, and finally scanned by a Digital Slide Scanner (Motic China Group Ltd.). The damage severity of colon section was evaluated and scored from 0 to 4 for ulceration of epithelium, crypt damage, depletion of goblet cells, edema, and inflammation by a pathologist in blinded fashion.

2.6. Biochemical Analysis of the Colon. Colon samples of a certain weight were homogenized in normal saline solution and then centrifuged at $12000 \mathrm{~g}\left(10 \mathrm{~min}\right.$ at $\left.4^{\circ} \mathrm{C}\right)$. The supernatant was used to determine the total protein concentration using a BCA protein assay kit (Beyotime Biotechnology, Shanghai, China). The contents of IL-6, IL-10, and TNF- $\alpha$ in the colon supernatant were determined using commercially available enzyme-linked immunosorbent assay kits (R\&D Systems, Minneapolis, MN, USA).

2.7. Gene Expression Analysis. Total RNA isolation from the colon tissue was performed using a FastPure Cell/Tissue Total RNA Isolation Kit (Vazyme Biotech Co., Ltd., Nanjing, China), and then a RevertAid First Strand cDNA Synthesis Kit (Vazyme Biotech Co., Ltd., Nanjing, China) was used for cDNA synthesis. Real-time quantitative polymerase chain reaction (RT-qPCR) was performed using $\beta$-actin as an internal control to identify the expressions of mucin2 (MUC2), ZO-1, claudin-1, and occludin [41]. RT-qPCR was carried out on a CFX96 Real-Time System (Bio-Rad, Hercules, CA) using SYBR Green super mix (Bio-Rad, Hercules, CA) under the following program: $2 \mathrm{~min}$ at $95^{\circ} \mathrm{C}$, 39 cycles of $30 \mathrm{~s}$ at $95^{\circ} \mathrm{C}, 30 \mathrm{~s}$ at $60^{\circ} \mathrm{C}$, and $30 \mathrm{~s}$ at $72^{\circ} \mathrm{C}$. The $2^{-\Delta \Delta \mathrm{Cq}}$ method was used to analyze the results. Table 1 lists the sequences of primers used in this study.

2.8. Fecal DNA Extraction, Sequencing, and Analysis. The FastDNA Spin Kit for Feces (MP Biomedicals) was used to extract bacterial DNA from the stool samples of the mice. The gut microbiota genomes were sequenced according to the method described in a previous study [42]. Briefly, after amplification and purification, the DNA amplicons of the 16S rRNA sequences (V3-V4 region) in the bacterial DNA were sequenced by the MiSeq Illumina platform.

Principle coordinate analysis was performed to evaluate the beta diversity of the microbial communities. Microbial diversity was estimated by the Chao-1 index. LEfSe analysis was used to determine the intergroup differences in the fecal microbiota composition. 


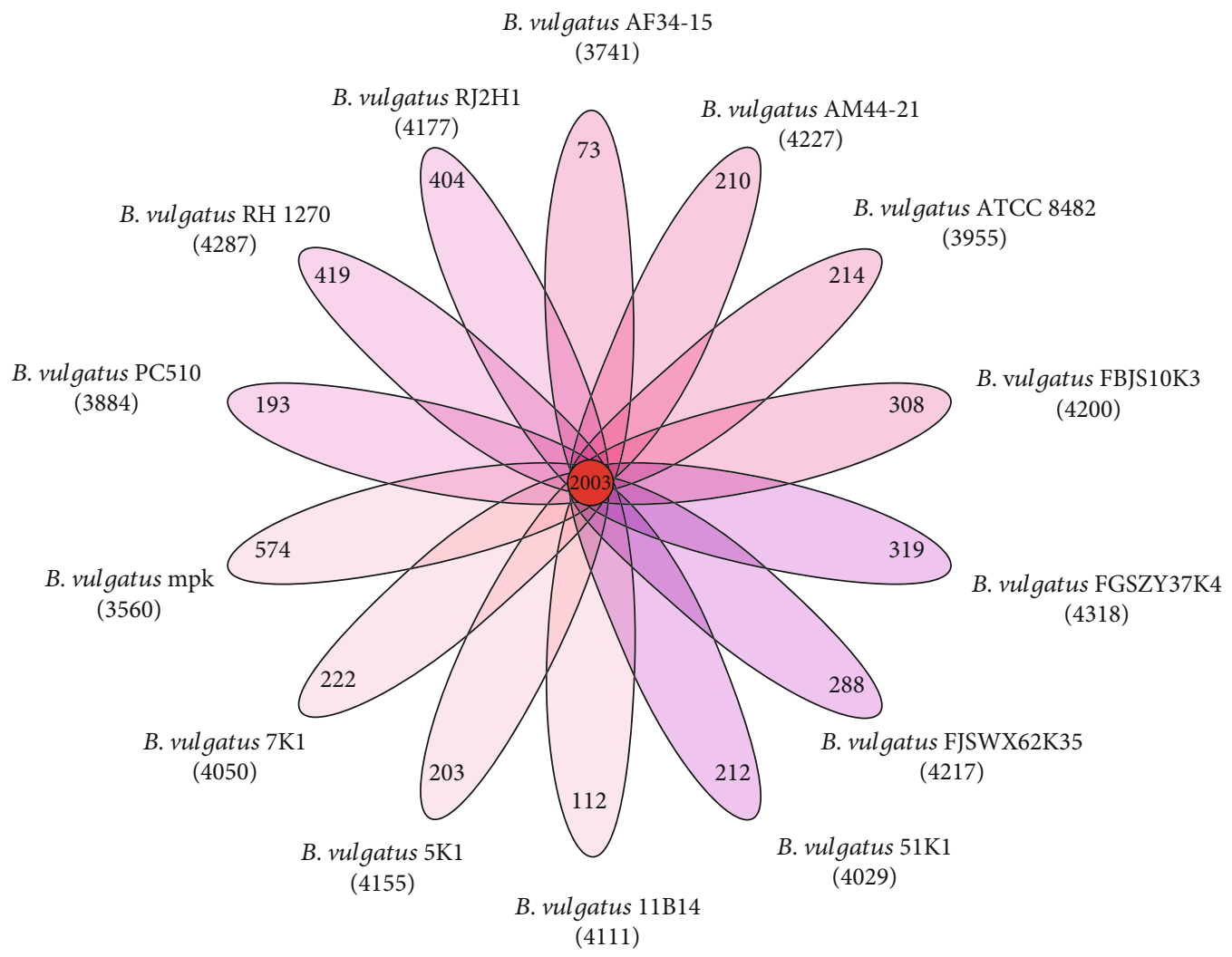

(a)

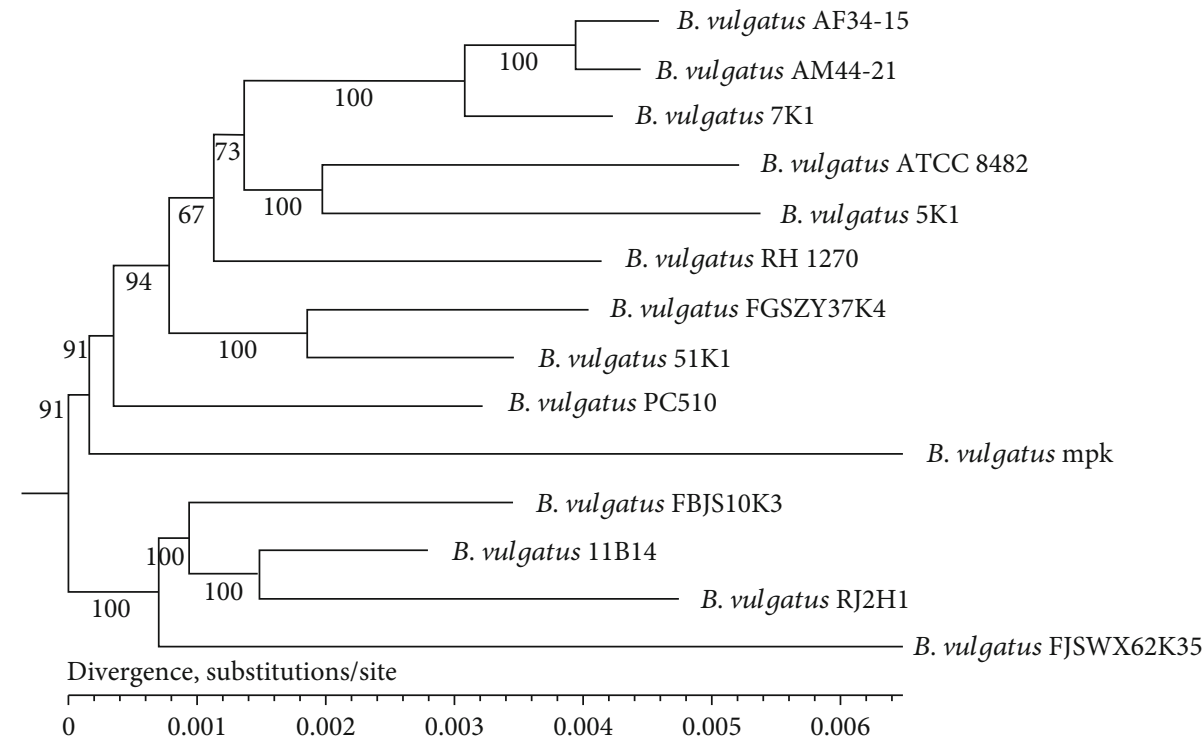

(b)

Figure 1: Core genes and phylogenetic analyses of 14 Bacteroides vulgatus strains. (a) Venn diagram of homologous clusters shared among the core genes. (b) Phylogenetic tree of 14 strains of B. vulgatus. Bootstrap confidence values were marked in the phylogenetic tree.

2.9. Determination of Short-Chain Fatty Acids (SCFAs) in Feces. To extract SCFAs (acetate, propionate, isobutyrate, butyrate, valerate, and isovalerate), the fecal samples were weighed, then soaked in saturated $\mathrm{NaCl}$ solution, acidified with sulfuric acid (10\%), and treated with diethyl ether. Gas chromatography-mass spectrometry (GC-MS) was then per- formed to determine the SCFAs concentrations in the feces, as described in [43]. Briefly, helium was used as the carrier gas with a flow rate of $2 \mathrm{~mL} / \mathrm{min}$, and injection volume was $1 \mu \mathrm{L}$ at an injection temperature of $240^{\circ} \mathrm{C}$. The following GC-MS temperature program was used: initial temperature $100^{\circ} \mathrm{C}$, increase to $140^{\circ} \mathrm{C}$ at $7.5^{\circ} \mathrm{C} / \mathrm{min}$, then rise to $200^{\circ} \mathrm{C}$ at 


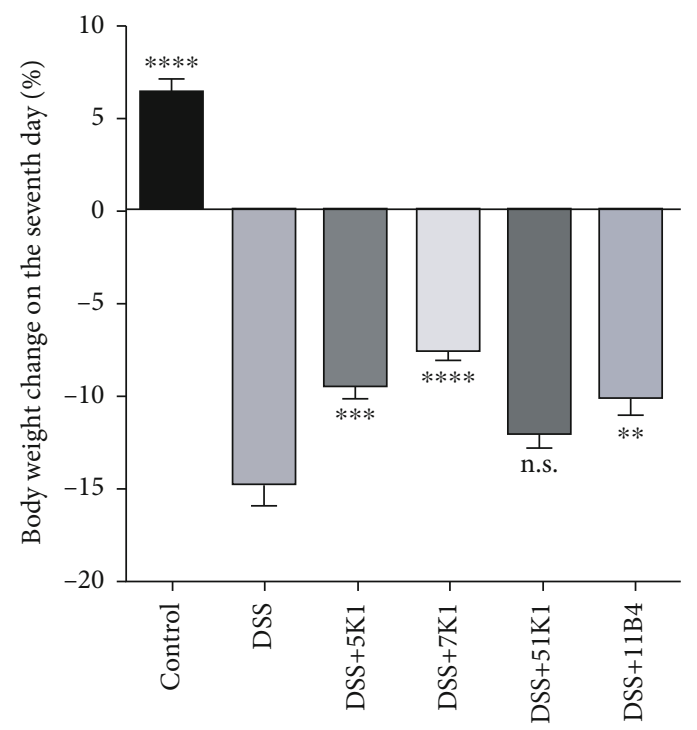

(a)

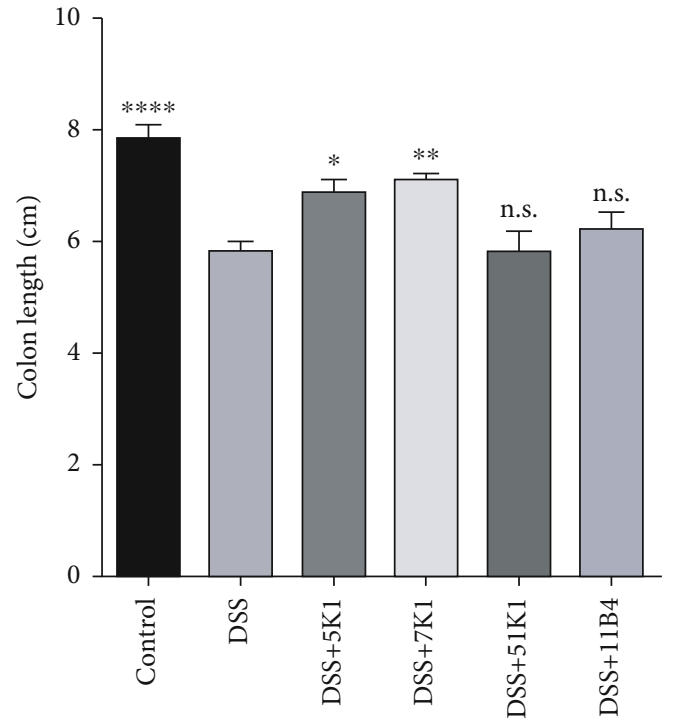

(b)

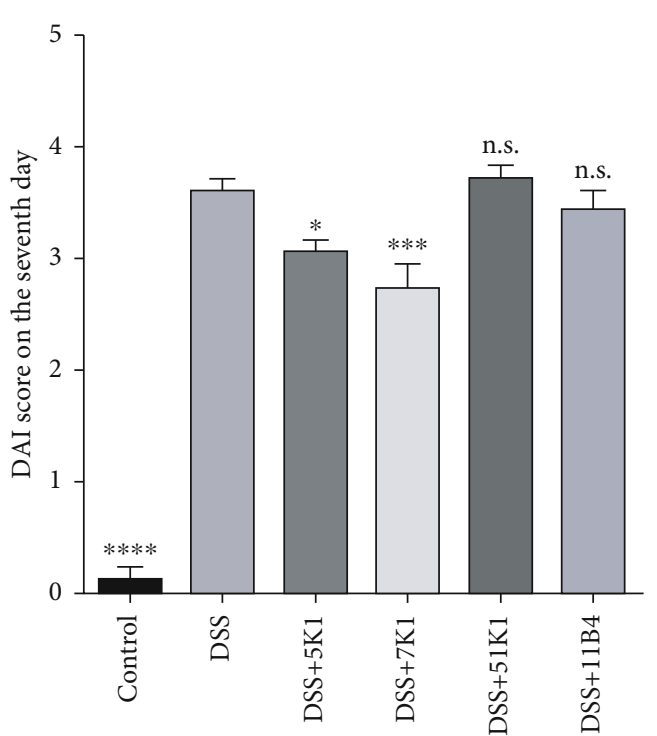

(c)

FIGURE 2: Effect of Bacteroides vulgatus on colitis symptoms. (a) Body weight. (b) Colon length. (c) Disease activity index (DAI). Six mice per group.

$60^{\circ} \mathrm{C} / \mathrm{min}$ with a hold time of $3 \mathrm{~min}$ and an ion source temperature of $220^{\circ} \mathrm{C}$. The external standard method was used to calculate the SCFA concentrations.

2.10. Statistical Analysis. All statistical analyses were performed using GraphPad Prism software version 6.0. The experimental data are expressed as the mean \pm the standard error of the mean. A one-way analysis of variance with Tukey's multiple comparison test was performed to determine the significance of the differences, and $p<0.05$ was considered to be statistically significant. The symbol $*$ indicates that the difference between the DSS group and the treated groups is significant, with $*, * *, * * *$, and $* * * *$ indicating $p<0.05, p<0.01, p<0.001$, and $p<0.0001$, respectively. The symbol n.s. indicates that the difference between the DSS group and other groups has no significance.

\section{Results}

3.1. Genetic Diversity and Evolution of B. vulgatus Strains. The 14 B. vulgatus strains (7 strains from this study and 7 strains from the NCBI database) shared 2003 orthologous genes (Figure 1(a)). The neighbor-joining tree established on the basis of these 2003 core genes shows that the strains are distributed into several branches (Figure 1(b)). We selected four strains $(5 \mathrm{~K} 1,7 \mathrm{~K} 1,51 \mathrm{~K} 1$, and $11 \mathrm{~B} 14)$ located far from each other on the phylogenetic tree to evaluate their effects on alleviating DSS-induced colitis in mice.

3.2. Effect of B. vulgatus on Colitis Symptoms. DSS exposure was found to markedly deteriorate the intestinal physiology of the mice, accompanied with weight loss, shortening of the colon length, and increased DAI scores (Figure 2). 
Notably, the administration of $B$. vulgatus strain $7 \mathrm{~K} 1$, but not $5 \mathrm{~K} 1,51 \mathrm{~K} 1$, or $11 \mathrm{~B} 14$, led to a significant recovery of these three physiological indicators.

3.3. Effect of B. vulgatus on the Intestinal Permeability of DSSTreated Mice. To estimate the effect of $B$. vulgatus strains on the intestinal permeability of the mice, we determined the serum FITC levels. After the DSS challenge, the FITC levels in the mice serum were markedly increased compared with those in the control group (Figure 3). These levels were markedly reduced in the mice fed with $B$. vulgatus $5 \mathrm{~K} 1$ or $B$. vulgatus $7 \mathrm{~K} 1$. However, neither B. vulgatus $51 \mathrm{~K} 1$ nor B. vulgatus $11 \mathrm{~B} 14$ showed any intestinal protective effect.

3.4. Effect of B. vulgatus on DSS-Induced Colonic Tissue Injury. The colon tissues of the mice in the DSS group, as compared to the control group, exhibited severe inflammatory cell infiltration, submucosal edema, significant disappearance of goblet cells, and severe damage to the epithelial structure (Figure 4(a)). The histological scores are a quantifiable indication of colonic injury. Compared with the DSS-treated mice, the colon tissue damage, expressed as the integrity of the intestinal epithelium and the alleviation of submucosal edema, in the mice fed with $B$. vulgatus $7 \mathrm{~K} 1$ was significantly reduced (Figure 4(b)), whereas it was obviously aggravated in the mice fed with $B$. vulgatus $5 \mathrm{~K} 1,11 \mathrm{~B} 14$, or $51 \mathrm{~K} 1$.

3.5. Effect of B. vulgatus on the Secretion of Inflammatory Factors in DSS-Treated Mice. Treatment with DSS resulted in dramatic alterations in the colonic immunomodulatory indicators of the mice, including increases in the concentrations of the proinflammatory cytokines TNF- $\alpha$ and IL-6, and a decrease in the concentration of the anti-inflammatory cytokine IL-10 (Figure 5). Among the tested strains, B. vulgatus $7 \mathrm{~K} 1$ was the most effective in restoring the expression of the three inflammatory cytokines by significantly inhibiting the increases in TNF- $\alpha$ and IL- 6 concentrations and upregulating the IL-10 concentrations up to those found in the control group. However, apart from reducing the IL- 6 concentrations (Figure 5(b)), B. vulgatus 5K1 induced no alterations in any of the other indicators. In addition, $B$. vulgatus $51 \mathrm{~K} 1$ significantly increased the TNF- $\alpha$ concentrations (Figure 5(a)).

3.6. Comparative Genomic Analysis of the Specific Genes in Different B. vulgatus Strains. We performed COG annotation to predict the functional genes of B. vulgatus $7 \mathrm{~K} 1$ and $B$. vulgatus $51 \mathrm{~K} 1$, and we found that $30 \mathrm{COG}$ families were present only in the B. vulgatus $7 \mathrm{~K} 1$ genome (Table S1). Except for three genes assigned to the COG category "General function prediction only" and four assigned to the COG category "Function unknown," most of the genes were mainly related to metabolism, transport, replication, recombination, repair, and defense. In addition, according to the annotation results from the CAZy database, the abundance of genes from 14 glycoside hydrolase families (GH3, GH5, GH15, GH20, GH33, GH43_24, GH141, GH95, GH105, GH29, GH106, GH27, GH99, and GH109) and 3 glycosyl transferase families (GT28, GT6, and GT4) was relatively high in the $B$. vulgatus $7 \mathrm{~K} 1$ genome (Table S2).

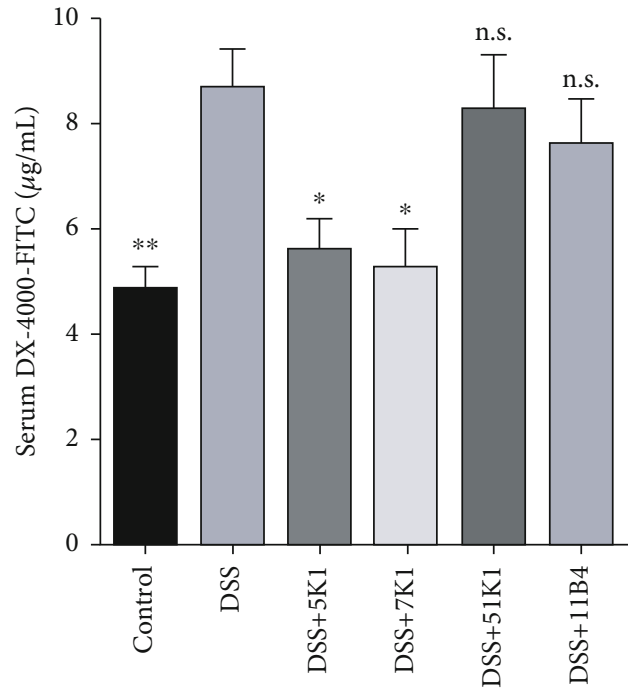

Figure 3: Effects of Bacteroides vulgatus on the intestinal permeability of mice. Four mice per group.

3.7. Effect of B. vulgatus on SCFA Concentrations in Feces. The concentrations of isobutyrate, valerate, and isovalerate in the fecal samples were not significantly different in the DSS group compared with the control group (Figure 6). Notably, after the oral administration of $B$. vulgatus $7 \mathrm{~K} 1$, the concentrations of these SCFAs markedly increased. Furthermore, the concentrations of acetate, propionate, and butyrate were significantly decreased in the DSS group compared with the control group. Treatment with $B$. vulgatus $7 \mathrm{~K} 1$ dramatically restored the acetate and butyrate concentrations close to their concentrations in the control group, but did not result in a significant change in the propionate concentrations.

3.8. Effect of B. vulgatus on the mRNA Levels of Genes Related to the Intestinal Barrier in Colon Tissue. To assess the intestinal mucosal barrier of the mice, we measured the relative expression levels of genes related to TJ proteins (ZO-1, occludin, and claudin-1) and MUC2. The results showed that the DSS treatment significantly decreased the expression of these four mucosal barrier indicators (Figure 7). Notably, oral gavage of $B$. vulgatus $7 \mathrm{~K} 1$ played a protective role against DSS-induced alterations in ZO-1 and claudin-1 expression. Although the expression of occludin and MUC2 was upregulated by $B$. vulgatus $7 \mathrm{~K} 1$, the results were not statistically significant.

3.9. Effect of B. vulgatus on the Composition of the Bacterial Community. Compared with the control group, DSS treatment was found to affect the composition of the gut microbiota (Figure $8(\mathrm{a})$ ) and slightly decrease the microbial diversity (Figure $8(\mathrm{~b})$ ), although these results were not statistically significant. Cotreatments with DSS and $B$. vulgatus $7 \mathrm{~K} 1$ induced similar results. At the genus level, $B$. vulgatus $7 \mathrm{~K} 1$ treatment markedly increased the abundance of Turicibacter and Romboutsia in comparison with that in the DSS group (Figure 8(c)). 


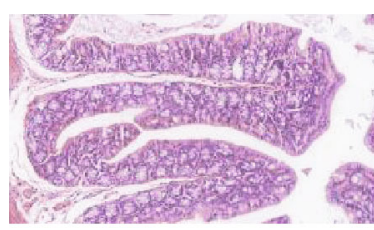

Control

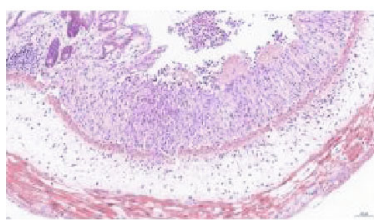

$\mathrm{DSS}+5 \mathrm{~K} 1$

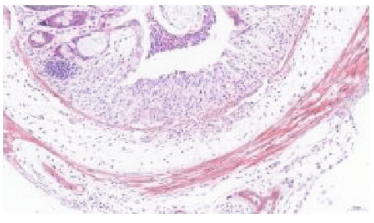

DSS $+51 \mathrm{~K} 1$

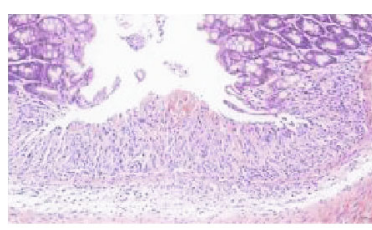

DSS

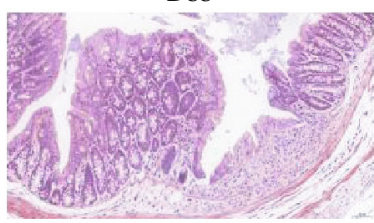

$\mathrm{DSS}+7 \mathrm{~K} 1$

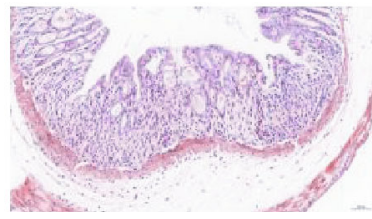

DSS+11B14

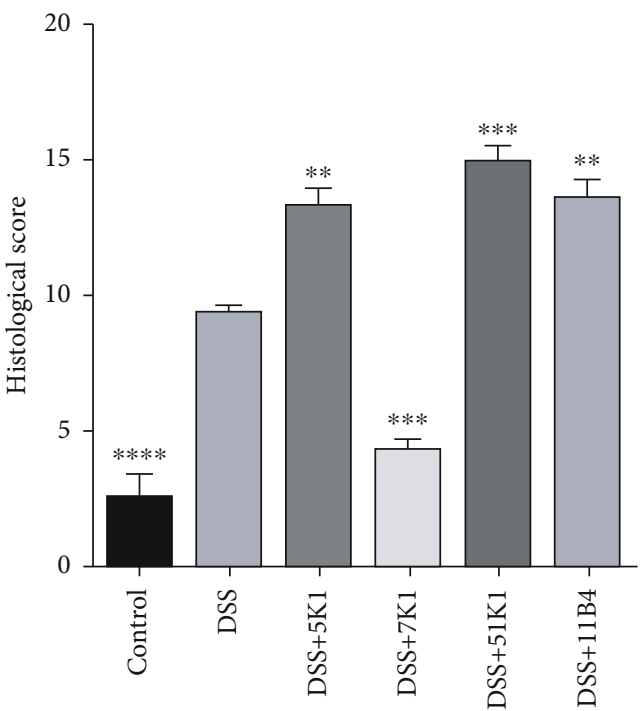

(a)

(b)

FIGURE 4: Effects of Bacteroides vulgatus on colonic tissue injury in mice. (a) Histological images. (b) Histological score. Four mice for the control group and three mice for each of the other groups.

\section{Discussion}

Numerous studies have shown that different strains of probiotics have different anticolitis effects [44-46]. B. vulgatus, a next-generation probiotic, has also been found to prevent colitis, depending on the strain [33]. Many factors influence the strain-specific effects of probiotics. The survivability of probiotics during transit through the gastrointestinal tract directly affects their abundance in the gut [47]. Several studies have shown that the protective effects of probiotic strains on the host health are dose-dependent [48-50]. Research has also revealed the enhanced ability of microencapsulated Lactobacillus rhamnosus GG to tolerate the stomach and small intestine environments, which can strengthen its efficacy in ameliorating the symptoms of colitis [51]. Hence, the ability of probiotics to resist the harsh environment of the gastrointestinal tract is a crucial factor that influences their colitisameliorating effects. Pathogenic bacteria such as Citrobacter rodentium and enterohemorrhagic $E$. coli can attach themselves to intestinal epithelial cells and then activate an immune response in the gut that can cause severe colitis $[52,53]$. Hence, the ability of probiotics to inhibit pathogen colonization in the intestine is essential to their effectiveness against colitis. A study has revealed that the oral administration of Lactobacillus acidophilus can decrease the colonization and translocation of $C$. rodentium and then inhibit $C$. rodentium-induced colitis [54]. In addition, some probiotics can release antimicrobial factors such as hydrogen peroxide and bacteriocins, which can kill the pathogenic bacteria or inhibit their growth $[55,56]$. Enhancement of the intestinal epithelial barrier function by some probiotics is also directly related to their alleviation effect on colitis $[57,58]$. SCFAs, which are produced by certain probiotics, can promote mucin expression [59] or stimulate the expression of TJ proteins [60], which serve to maintain the intestinal integrity [61]. These findings suggest that the different anticolitis abilities of probiotic strains are attributable to their complex physiological characteristics.

Genomic diversity implies functional specificity. Several Lactobacillus fermentum strains with considerable genomic differences have been reported to exhibit different antiinflammatory effects on colitis in mice [46]. Hence, we constructed an evolutionary tree and selected four B. vulgatus strains that have large genomic differences. We then assessed their efficacy in ameliorating DSS-induced colitis in mice. Our results showed that among the four B. vulgatus strains, only $B$. vulgatus $7 \mathrm{~K} 1$ could significantly relieve five DSSinduced symptoms, including reduced body weight, a shortened colon, increased DAI scores, severe damage to the colon tissue, and increased intestinal permeability. An abnormal immune response is an important indicator of the pathogenesis of colitis. Proinflammatory cytokines TNF- $\alpha$ and IL- 6 have been reported to result in mucosal inflammation and aggravate immune disorders $[62,63]$. Reducing TNF- $\alpha$ and IL-6 in mice with colitis was considered to be a logical target for the treatment of colitis [64]. The experiment in IL-10deficient mice has proved the vital role of IL-10 in preventing IBD [65]. As an anti-inflammatory cytokine, IL-10 has been reported to inhibit the expression of TNF- $\alpha$ in immune regulatory processes [66]. Moreover, the protective effects of $B$. fragilis NCTC 9343 against colitis induced by trinitrobenzene sulphonic acid (TNBS) or Helicobacter hepaticus largely attributed to its ability to increase the production of IL-10 $[8,22]$. In our study, the oral administration of B. vulgatus 


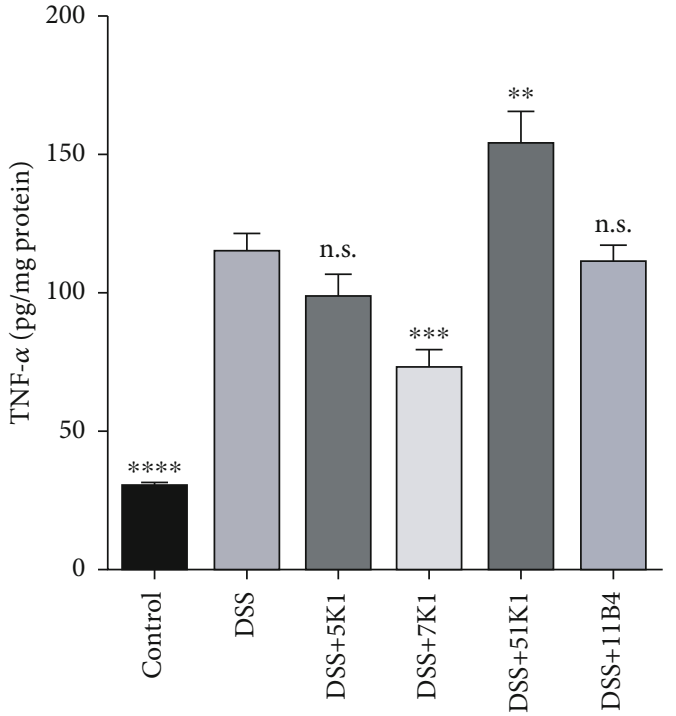

(a)

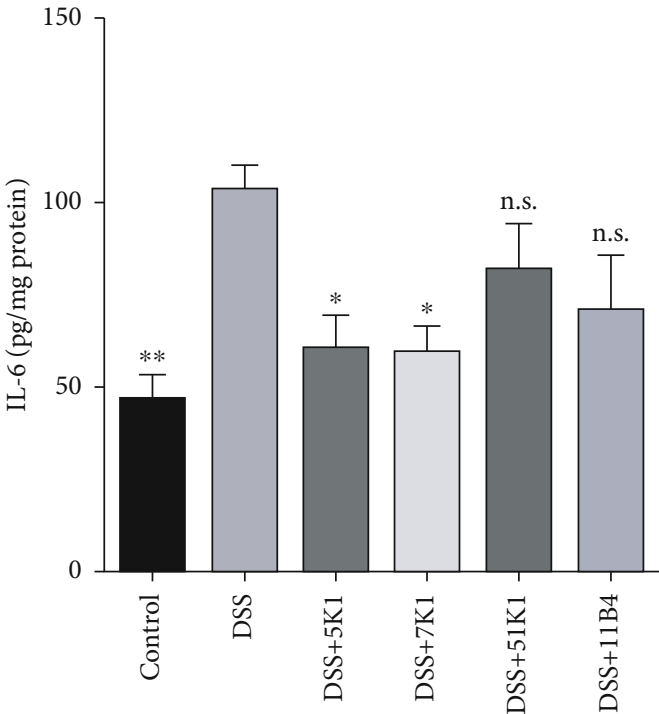

(b)

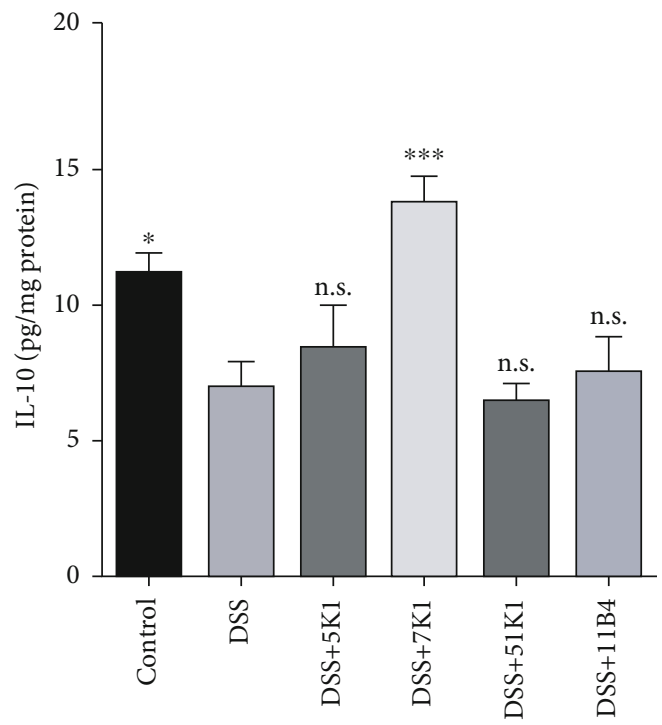

(c)

Figure 5: Effects of Bacteroides vulgatus on the concentrations of inflammatory cytokines. (a) TNF- $\alpha$. (b) IL-6. (c) IL-10. Six mice per group.

$7 \mathrm{~K} 1$, rather than $B$. vulgatus $5 \mathrm{~K} 1$, not only significantly reduced the concentrations of TNF- $\alpha$ and IL- 6 but also markedly increased the production of IL-10 in the colon tissue of mice. Hence, given these results, we found B. vulgatus $7 \mathrm{~K} 1$ to be significantly more effective in relieving DSSinduced colitis in mice than the other $B$. vulgatus strains tested in this study. We note that B. vulgatus $51 \mathrm{~K} 1$ was the only one of the four strains that failed to restore the weight loss, shortened colon, and increased DAI scores caused by DSS. Furthermore, B. vulgatus $51 \mathrm{~K} 1$, but not the other three strains, significantly increased both the tissue damage and TNF- $\alpha$ concentration in the mouse colons, as compared to the DSS group. These results indicate that $B$. vulgatus $51 \mathrm{~K} 1$ can dramatically aggravate colitis.

Subsequently, we performed a comparative genomic analysis to understand the differences in the anti-inflammatory effects of $B$. vulgatus $7 \mathrm{~K} 1$ and $B$. vulgatus $51 \mathrm{~K} 1$. Among the
30 strain-specific COGs for B. vulgatus 7K1, COG2977 is involved in secondary metabolite biosynthesis, transport, and catabolism and may be related to the production of SCFAs. Moreover, GH43_24, which is more abundant in the B. vulgatus $7 \mathrm{~K} 1$ genome than in the $B$. vulgatus $51 \mathrm{~K} 1$ genome, is mainly responsible for the hydrolysis of xylan. A previous study reported that feeding mice xylooligosaccharides can increase the production of fecal SCFAs [67]. In our experiments, $B$. vulgatus $7 \mathrm{~K} 1$ significantly promoted the production of SCFAs, including acetate, butyrate, isobutyrate, valerate, and isovalerate (Figure 6).

The protective role of SCFAs, especially butyrate, acetate, and propionate, in intestinal inflammatory diseases has been widely demonstrated and is well recognized. Mechanistically, SCFAs may promote the integrity of the intestinal epithelial barrier by increasing the synthesis of mucins in the mucosal layer [68] and of TJ proteins in the epithelial monolayer 


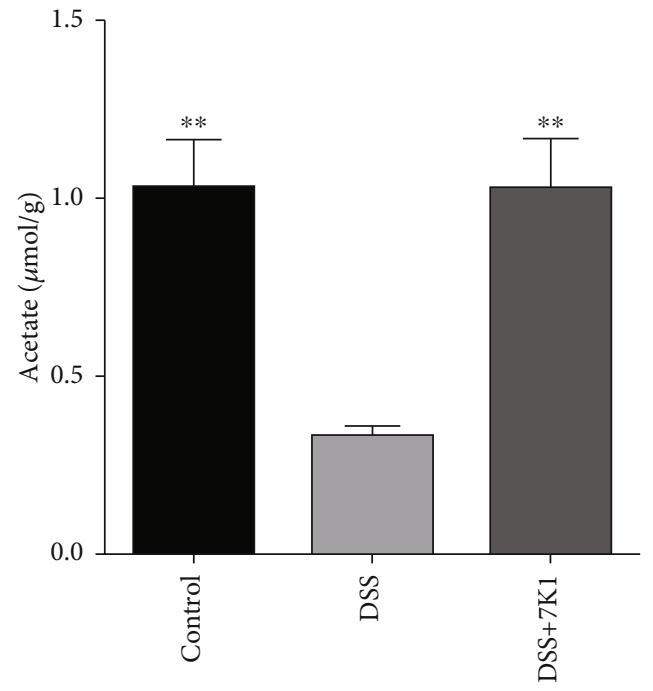

(a)

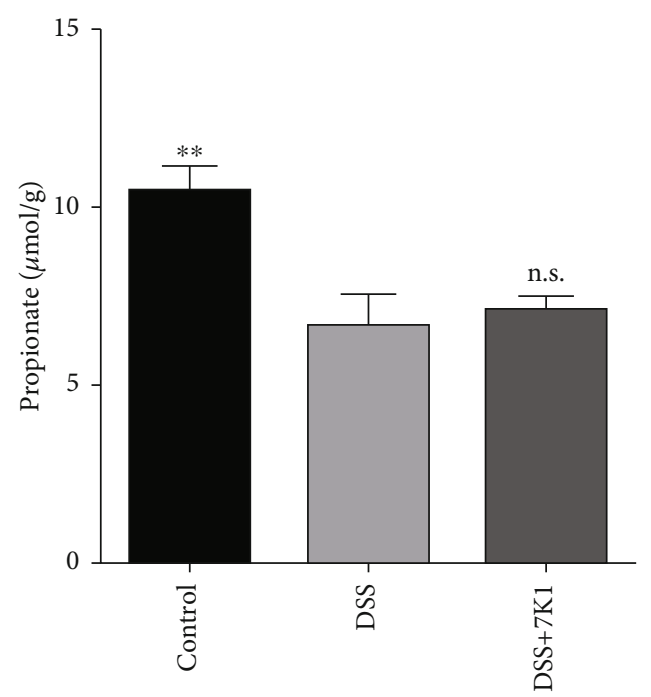

(c)

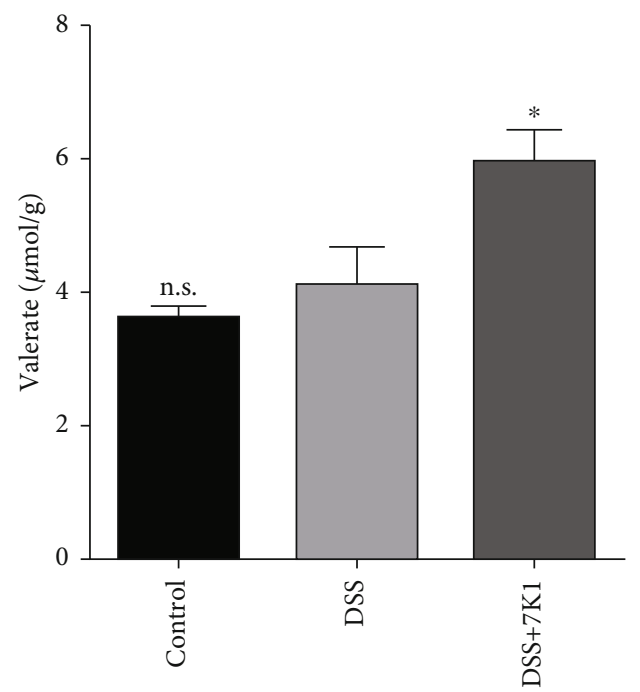

(e)

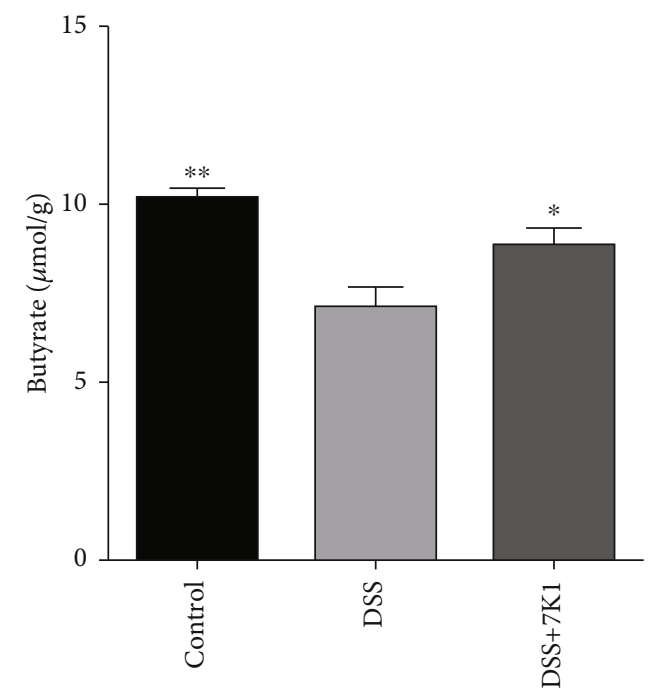

(b)

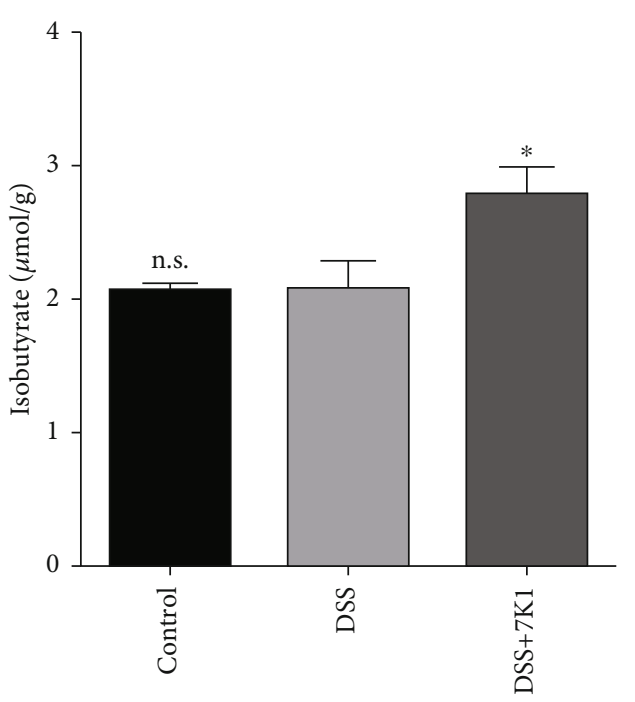

(d)

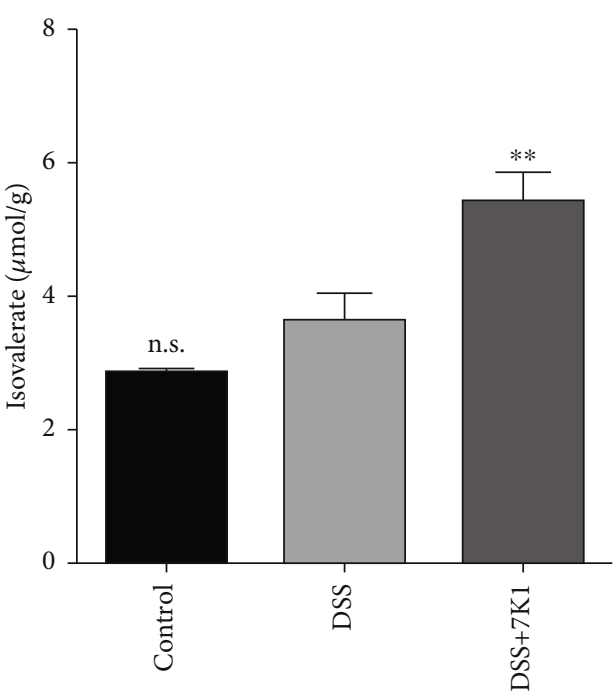

(f)

Figure 6: Effects of Bacteroides vulgatus on the concentrations of short-chain fatty acids in the fecal samples of mice. (a) Acetate. (b) Propionate. (c) Butyrate. (d) Isobutyrate. (e) Valerate. (f) Isovalerate. Five mice per group. 


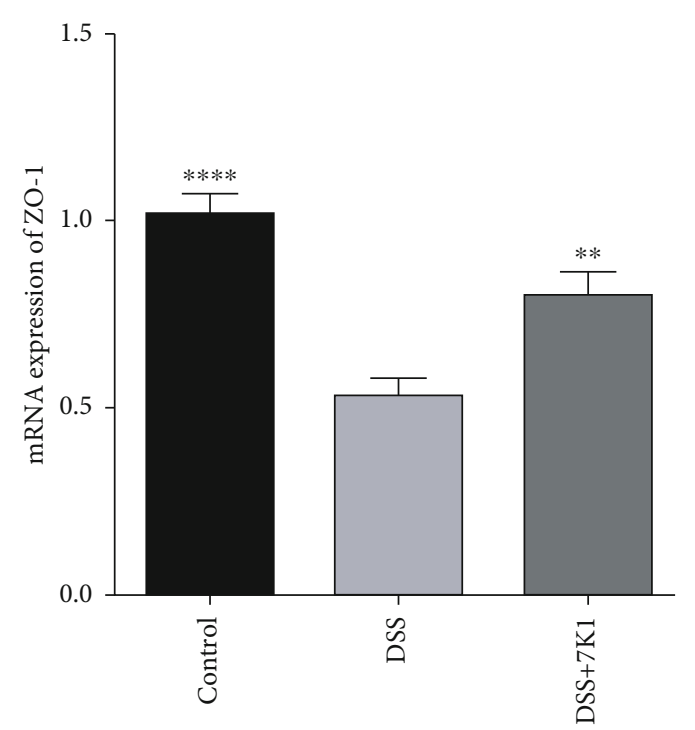

(a)

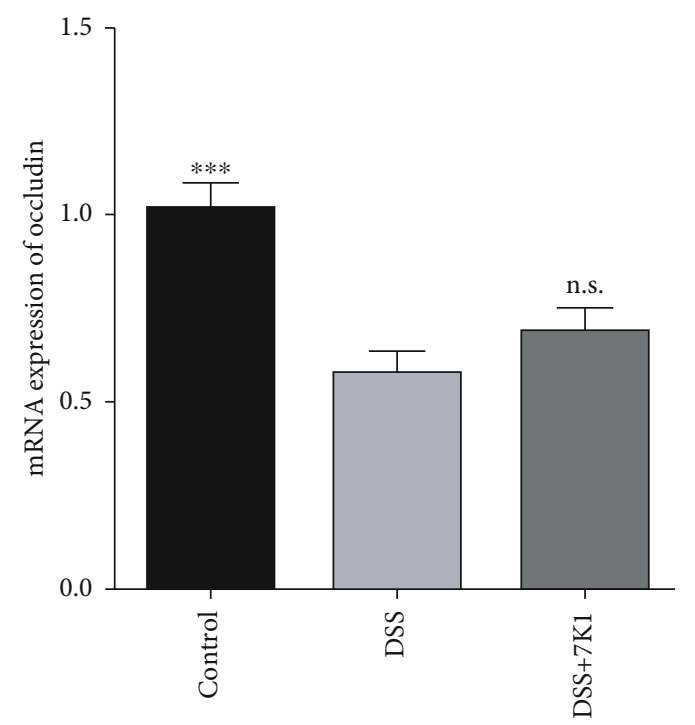

(c)

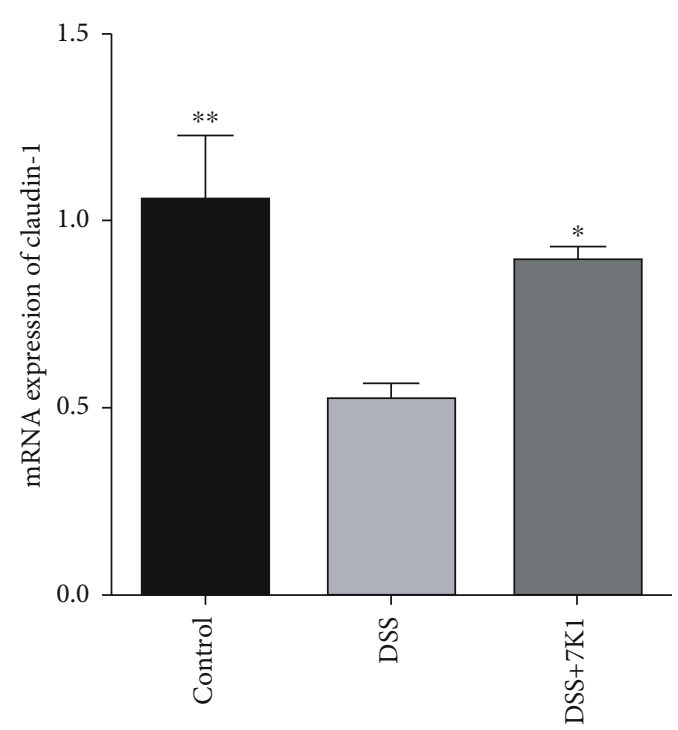

(b)

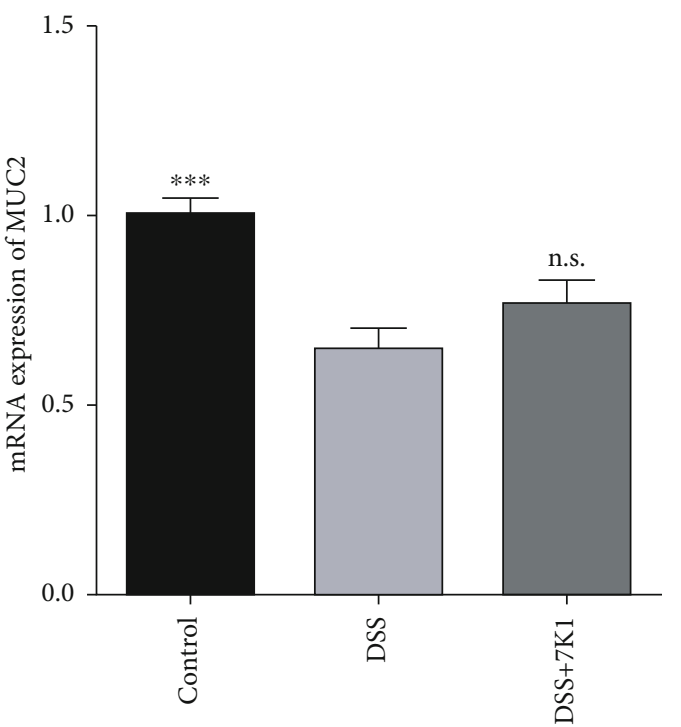

(d)

Figure 7: Effects of Bacteroides vulgatus on the intestinal barrier of mice. (a) ZO-1. (b) Claudin-1. (c) Occludin. (d) MUC2. Six mice per group.

$[69,70]$, and thus, contribute to the remission of colitis. In addition, SCFAs attenuate colitis in mice by restoring the balance of gut microbial dysbiosis [71, 72]. In this study, feeding $B$. vulgatus $7 \mathrm{~K} 1$ to mice protected the TJs of their intestinal epithelial cells (Figure 7), but did not restore the balance of the gut microbial dysbiosis caused by DSS (Figure 8). Hence, enhancing the integrity of the epithelial monolayer by increasing the SCFAs may be a protective mechanism of $B$. vulgatus $7 \mathrm{~K} 1$ against DSS-induced colitis.

COG4464, which is specific to $B$. vulgatus $7 \mathrm{~K} 1$, is responsible for the biosynthesis of capsular polysaccharides. The anti-inflammatory effect of the capsular polysaccharide produced by certain Bacteroides strains has been reported in several studies $[5,10]$. As the best-studied zwitterionic capsular polysaccharide, polysaccharide A has been confirmed to prevent colitis by inducing the expression of IL-10 in the colon [73]. Our results showed that B. vulgatus $7 \mathrm{~K} 1$, but not $B$. vulgatus $51 \mathrm{~K} 1$, markedly upregulated the IL-10 expression in the mouse colons (Figure 5). Thus, the gene belonging to COG4464 may partly account for the anti-inflammatory property of $B$. vulgatus $7 \mathrm{~K} 1$.

The specific genes of $B$. vulgatus $7 \mathrm{~K} 1$, denoted as COG0270, COG1343, and COG3392, are responsible for DNA replication, recombination, and repair and are integral to cell survival [74]. In addition, another specific gene, denoted as COG0610, belonging to B. vulgatus $7 \mathrm{~K} 1$ is related to the type I site-specific restriction-modification system, which has been found to protect bacteria from bacteriophage infection [75]. Bacteriophages are members of the host gut 


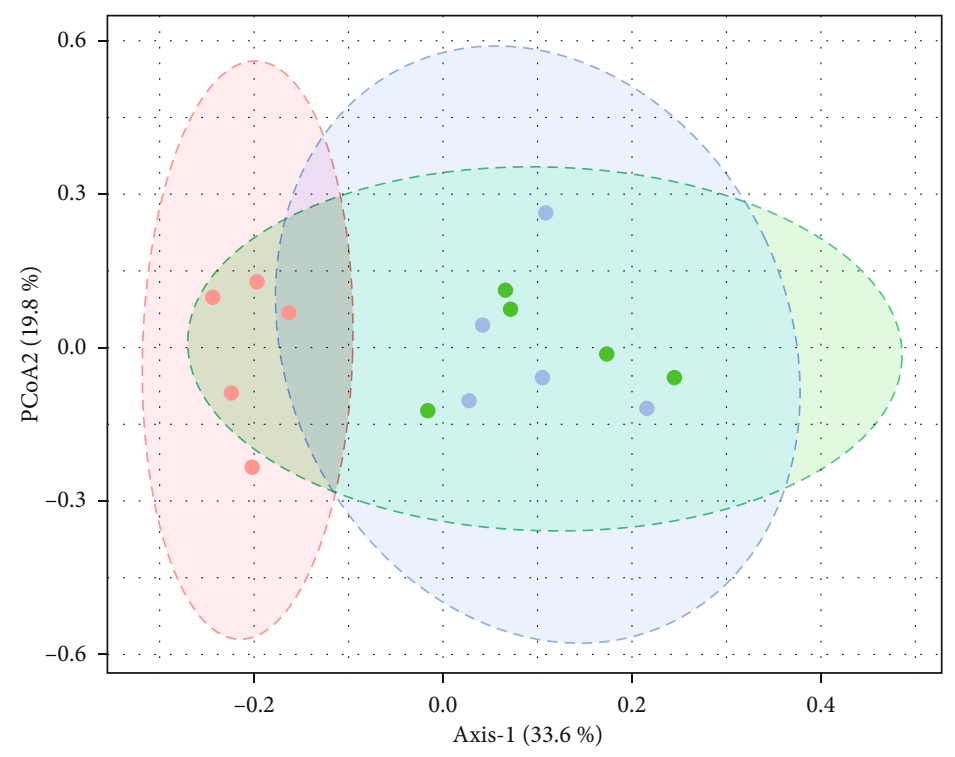

Sample type

- Control

- DSS

- DSS+7K1

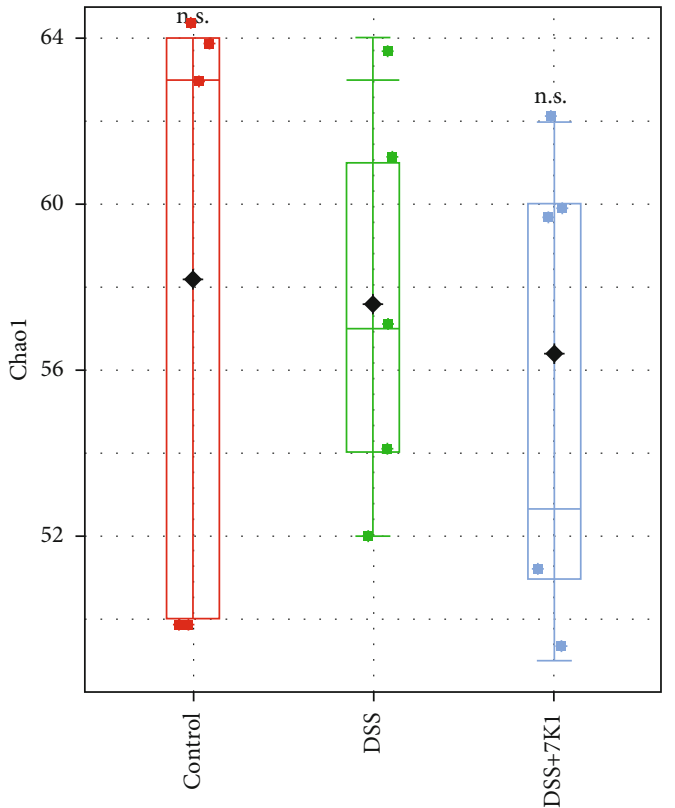

Class

Control

DSS

DSS+7K1

(a)

(b)

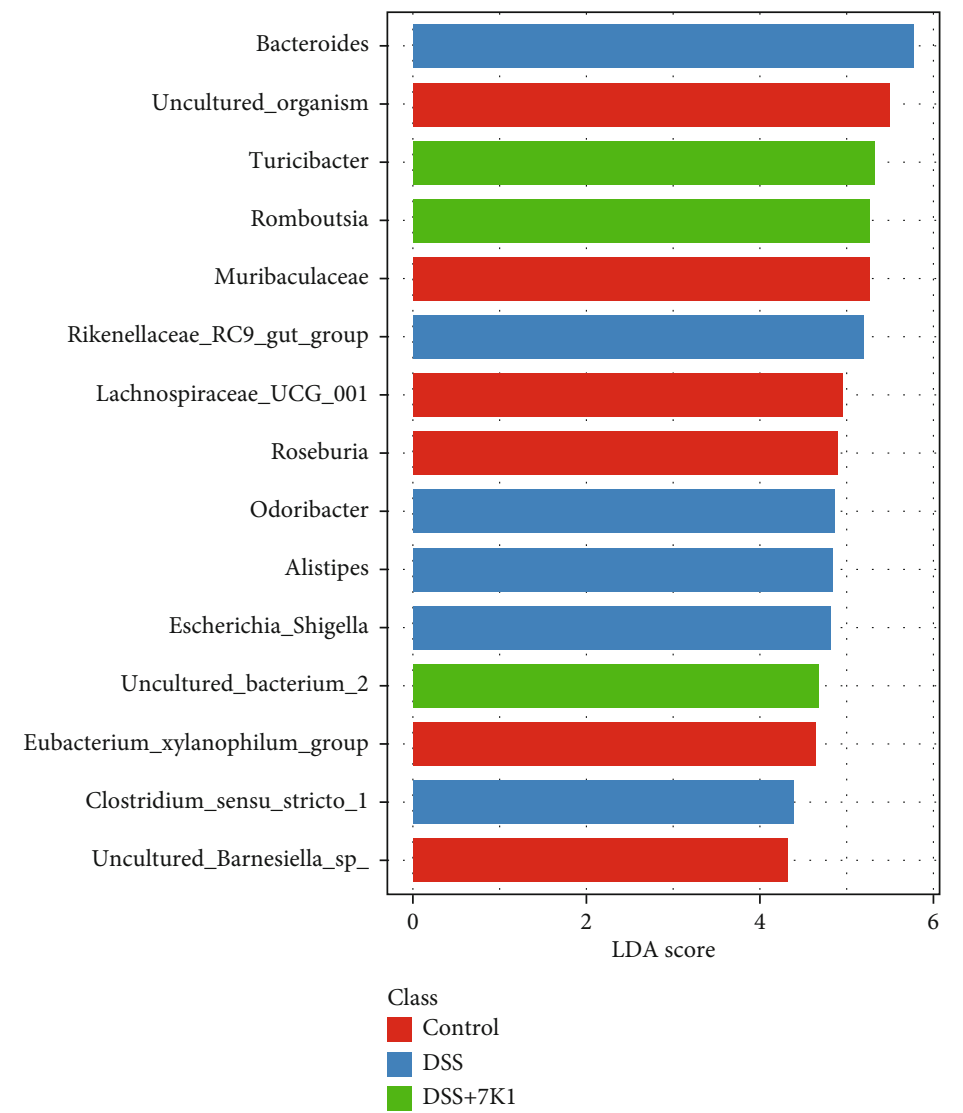

(c)

Figure 8: Effect of Bacteroides vulgatus on the intestinal microbiota of mice. (a) Principal component analysis of gut microbiota. (b) Alpha diversity indicated by Chaol index. (c) LEfSe analysis of the different groups. Five mice per group. 
microbiota. Environmental stimuli have been reported to induce the production of infectious bacteriophages that cause lysis in their bacterial host [76]. Hence, these genes may guarantee the survival of $B$. vulgatus $7 \mathrm{~K} 1$ in the gut of DSS-treated mice and further ensure its protective role in the host health.

Compared with $B$. vulgatus $51 \mathrm{~K} 1, B$. vulgatus $7 \mathrm{~K} 1$ has more gene copy numbers for the families GH29, GH95, and GH141. The $\alpha$-L-fucosidases of these glycoside hydrolase families are involved in the synthesis of fucosyl-Nacetylglucosamine disaccharides [77]. It has been reported that some fucosyl-N-acetylglucosamine disaccharides inhibit the adhesion of certain enteropathogenic E. coli (EPEC) strains onto HT29 cells [78]. EPEC adherence onto intestinal epithelial monolayers can disrupt the barrier function [79]. Thus, the greater number of genes of these glycoside hydrolase families in $B$. vulgatus $7 \mathrm{~K} 1$ may guarantee its protective effect on the intestinal barrier function.

\section{Conclusion}

The results of this study revealed that the protective roles of $B$. vulgatus strains selected from different clades of the phylogenetic tree against DSS-induced colitis are strain-specific. $B$. vulgatus $7 \mathrm{~K} 1$ exhibited a significant protective effect against colitis, but $B$. vulgatus $51 \mathrm{~K} 1$ markedly deteriorated the symptoms of colitis in mice. The results of further genomic comparisons showed that several specific genes present in the $B$. vulgatus 7K1 genome that are responsible for colonic SCFAs or capsular polysaccharide production and survival in the gut do not exist in the $B$. vulgatus $51 \mathrm{~K} 1$ genome. This may explain the effective protection provided by $B$. vulgatus $7 \mathrm{~K} 1$ against DSS-induced colitis, and the lack thereof by $B$. vulgatus $51 \mathrm{~K} 1$.

\section{Data Availability}

The data used to support the findings of this study are available from the corresponding author upon request.

\section{Conflicts of Interest}

The authors declare no conflicts of interest.

\section{Acknowledgments}

This work was supported by the National Natural Science Foundation of China [32001665], the Natural Science Foundation of Jiangsu Province [BK20180603], the National firstclass discipline program of Food Science and Technology [JUFSTR20180102], and Collaborative Innovationcenter of Food Safety and Quality Control in Jiangsu Province.

\section{Supplementary Materials}

Table S1: specific clusters of orthologous group (COG) categories in Bacteroides vulgatus 7K1. Table S2: details of the Carbohydrate-Active enZyme genes that are abundant in the genome of Bacteroides vulgatus 7K1. (Supplementary Materials)

\section{References}

[1] R. Liu, J. Hong, X. Xu et al., "Gut microbiome and serum metabolome alterations in obesity and after weight- loss intervention," Nature Medicine, vol. 23, no. 7, pp. 859-868, 2017.

[2] J. Y. Yang, Y. S. Lee, Y. Kim et al., "Gut commensal Bacteroides acidifaciens prevents obesity and improves insulin sensitivity in mice," Mucosal Immunology, vol. 10, no. 1, pp. 104-116, 2017.

[3] W. Zhang, B. Zhu, J. Xu et al., "Bacteroides fragilis protects against antibiotic-associated diarrhea in rats by modulating intestinal defenses," Frontiers in Immunology, vol. 9, p. 1040, 2018.

[4] C. Ramakrishna, M. Kujawski, H. Chu, L. Li, S. K. Mazmanian, and E. M. Cantin, "Bacteroides fragilis polysaccharide A induces IL-10 secreting B and T cells that prevent viral encephalitis," Nature Communications, vol. 10, no. 1, article 2153, 2019.

[5] L. Zheng, M. Luo, G. Kuang et al., "Capsular polysaccharide from Bacteroides fragilis protects against ulcerative colitis in an undegraded form," Frontiers in Pharmacology, vol. 11, article 570476, 2020.

[6] K. Takahashi, A. Nishida, T. Fujimoto et al., "Reduced abundance of butyrate-producing bacteria species in the fecal microbial community in Crohn's disease," Digestion, vol. 93, no. 1, pp. 59-65, 2016.

[7] W. Zhong, X. Lu, H. Shi et al., "Distinct microbial populations exist in the mucosa-associated microbiota of diarrhea predominant irritable bowel syndrome and ulcerative colitis," Journal of Clinical Gastroenterology, vol. 53, no. 9, pp. 660-672, 2019.

[8] S. K. Mazmanian, J. L. Round, and D. L. Kasper, "A microbial symbiosis factor prevents intestinal inflammatory disease," Nature, vol. 453, no. 7195, pp. 620-625, 2008.

[9] M. Delday, I. Mulder, E. T. Logan, and G. Grant, “Bacteroides thetaiotaomicron ameliorates colon inflammation in preclinical models of Crohn's disease," Inflammatory Bowel Diseases, vol. 25, no. 1, pp. 85-96, 2019.

[10] C. P. Neff, M. E. Rhodes, K. L. Arnolds et al., "Diverse intestinal bacteria contain putative zwitterionic capsular polysaccharides with anti-inflammatory properties," Cell Host \& Microbe, vol. 20, no. 4, pp. 535-547, 2016.

[11] E. C. Wick, S. Rabizadeh, E. Albesiano et al., "Stat3 activation in murine colitis induced by enterotoxigenic Bacteroides fragilis," Inflammatory Bowel Diseases, vol. 20, no. 5, pp. 821-834, 2014.

[12] R. Dziarski, S. Y. Park, D. R. Kashyap, S. E. Dowd, and D. Gupta, "Pglyrp-regulated gut microflora Prevotella falsenii, Parabacteroides distasonis and Bacteroides eggerthii enhance and Alistipes finegoldii attenuates colitis in mice," PLoS One, vol. 11, no. 1, article e0146162, 2016.

[13] M. Chatzidaki-Livanis, N. Geva-Zatorsky, and L. E. Comstock, "Bacteroides fragilis type VI secretion systems use novel effector and immunity proteins to antagonize human gut Bacteroidales species," Proceedings of the National Academy of Sciences of the United States of America, vol. 113, no. 13, pp. 3627-3632, 2016.

[14] M. Chatzidaki-Livanis, M. J. Coyne, K. G. Roelofs, R. R. Gentyala, J. M. Caldwell, and L. E. Comstock, "Gut symbiont Bacteroides fragilis secretes a eukaryotic-like ubiquitin protein that mediates intraspecies antagonism," MBio, vol. 8, no. 6, 2017. 
[15] N. T. Porter, P. Canales, D. A. Peterson, and E. C. Martens, "A subset of polysaccharide capsules in the human symbiont Bacteroides thetaiotaomicron promote increased competitive fitness in the mouse gut," Cell Host \& Microbe, vol. 22, no. 4, pp. 494-506.e8, 2017.

[16] A. L. Hecht, B. W. Casterline, Z. M. Earley, Y. A. Goo, D. R. Goodlett, and J. Bubeck Wardenburg, "Strain competition restricts colonization of an enteric pathogen and prevents colitis," EMBO Reports, vol. 17, no. 9, pp. 1281-1291, 2016.

[17] R. Corrêa-Oliveira, J. L. Fachi, A. Vieira, F. T. Sato, and M. A. R. Vinolo, "Regulation of immune cell function by short-chain fatty acids," Clinical \& Translational Immunology, vol. 5, no. 4, p. e73, 2016.

[18] R. B. Canani, M. Di Costanzo, and L. Leone, "The epigenetic effects of butyrate: potential therapeutic implications for clinical practice," Clinical Epigenetics, vol. 4, no. 1, p. 4, 2012.

[19] J. Sauer, K. K. Richter, and B. L. Pool-Zobel, "Physiological concentrations of butyrate favorably modulate genes of oxidative and metabolic stress in primary human colon cells," Journal of Nutritional Biochemistry, vol. 18, no. 11, pp. 736-745, 2007.

[20] M. H. Sofi, Y. Wu, T. Ticer et al., "A single strain of Bacteroides fragilis protects gut integrity and reduces GVHD," Jci Insight, vol. 6, no. 3, article e136841, 2021.

[21] R. J. Obiso, D. M. Lyerly, R. L. van Tassell, and T. D. Wilkins, "Proteolytic activity of the Bacteroides fragilis enterotoxin causes fluid secretion and intestinal damage in vivo," Infection and Immunity, vol. 63, no. 10, pp. 3820-3826, 1995.

[22] J. L. Round and S. K. Mazmanian, "Inducible Foxp $3^{+}$regulatory T-cell development by a commensal bacterium of the intestinal microbiota," Proceedings of the National Academy of Sciences of the United States of America, vol. 107, no. 27, pp. 12204-12209, 2010.

[23] Y. K. Lee, P. Mehrabian, S. Boyajian et al., "The protective role ofBacteroides fragilisin a murine model of colitis-associated colorectal Cancer," mSphere, vol. 3, no. 6, 2018.

[24] E. M. Brown, X. Ke, D. Hitchcock et al., "Bacteroides-derived sphingolipids are critical for maintaining intestinal homeostasis and symbiosis," Cell Host \& Microbe, vol. 25, no. 5, pp. 668680.e7, 2019.

[25] Q. Zhai, X. Shen, S. Cen et al., "Screening of Lactobacillus salivarius strains from the feces of Chinese populations and the evaluation of their effects against intestinal inflammation in mice," Food \& Function, vol. 11, no. 1, pp. 221-235, 2020.

[26] Y. Liu, Y. Li, X. Yu et al., "Physiological characteristics of Lactobacillus casei strains and their alleviation effects against inflammatory bowel disease," Journal of Microbiology and Biotechnology, vol. 31, no. 1, pp. 92-103, 2021.

[27] J. Qin, R. Li, J. Raes et al., "A human gut microbial gene catalogue established by metagenomic sequencing," Nature, vol. 464, no. 7285, pp. 59-65, 2010.

[28] J. Tap, S. Mondot, F. Levenez et al., "Towards the human intestinal microbiota phylogenetic core," Environmental Microbiology, vol. 11, no. 10, pp. 2574-2584, 2009.

[29] M. Waidmann, O. Bechtold, J. S. Frick et al., “Bacteroides vulgatus protects against Escherichia coli-induced colitis in gnotobiotic interleukin-2-deficient mice," Gastroenterology, vol. 125, no. 1, pp. 162-177, 2003.

[30] J. S. Frick, K. Fink, F. Kahl et al., "Identification of commensal bacterial strains that modulate Yersinia enterocolitica and dextran sodium sulfate-induced inflammatory responses: implica- tions for the development of probiotics," Infection and Immunity, vol. 75, no. 7, pp. 3490-3497, 2007.

[31] H. C. Rath, K. H. Wilson, and R. B. Sartor, "Differential induction of colitis and gastritis in HLA-B27 transgenic rats selectively colonized with Bacteroides vulgatus or Escherichia coli," Infection and Immunity, vol. 67, no. 6, pp. 2969-2974, 1999.

[32] A. B. Onderdonk, R. L. Cisneros, and R. T. Bronson, "Enhancement of experimental ulcerative colitis by immunization with Bacteroides vulgatus," Infection and Immunity, vol. 42, no. 2, pp. 783-788, 1983.

[33] A. B. Onderdonk, R. Bronson, and R. Cisneros, "Comparison of Bacteroides vulgatus strains in the enhancement of experimental ulcerative colitis," Infection and Immunity, vol. 55, no. 3, pp. 835-836, 1987.

[34] S. Wirtz, V. Popp, M. Kindermann et al., "Chemically induced mouse models of acute and chronic intestinal inflammation," Nature Protocols, vol. 12, no. 7, pp. 1295-1309, 2017.

[35] M. Murano, K. Maemura, I. Hirata et al., "Therapeutic effect of intracolonically administered nuclear factor kappa B (p65) antisense oligonucleotide on mouse dextran sulphate sodium (DSS)-induced colitis," Clinical and Experimental Immunology, vol. 120, no. 1, pp. 51-58, 2000.

[36] R. Q. Li, Y. R. Li, K. Kristiansen, and J. Wang, "SOAP: short oligonucleotide alignment program," Bioinformatics, vol. 24, no. 5, pp. 713-714, 2008.

[37] R. Q. Li, H. M. Zhu, J. Ruan et al., "De novo assembly of human genomes with massively parallel short read sequencing," Genome Research, vol. 20, no. 2, pp. 265-272, 2010.

[38] K. Katoh and D. M. Standley, "MAFFT multiple sequence alignment software version 7: improvements in performance and usability," Molecular Biology and Evolution, vol. 30, no. 4, pp. 772-780, 2013.

[39] J. S. Zheng, L. F. Ruan, M. Sun, and M. Gänzle, “A genomic view of Lactobacilli and Pediococci demonstrates that phylogeny matches ecology and physiology," Applied \& Environmental Microbiology, vol. 81, no. 20, pp. 7233-7243, 2015.

[40] Q. X. Zhai, F. W. Tian, J. X. Zhao, H. Zhang, A. Narbad, and W. Chen, "Oral administration of probiotics inhibits absorption of the heavy metal cadmium by protecting the intestinal barrier," Applied and Environmental Microbiology, vol. 82, no. 14, pp. 4429-4440, 2016.

[41] Q. Xu, X. F. Li, E. Y. Wang et al., "A cellular model for screening of Lactobacilli that can enhance tight junctions," RSC Advances, vol. 6, no. 113, pp. 111812-111821, 2016.

[42] L. L. Wang, M. L. Pan, D. Y. Li et al., "Metagenomic insights into the effects of oligosaccharides on the microbial composition of cecal contents in constipated mice," Journal of Functional Foods, vol. 38, pp. 486-496, 2017.

[43] B. Y. Mao, D. Y. Li, C. Q. Ai, J. Zhao, H. Zhang, and W. Chen, "Lactulose differently modulates the composition of luminal and mucosal microbiota in C57BL/6J mice," Journal of Agricultural and Food Chemistry, vol. 64, no. 31, pp. 6240-6247, 2016.

[44] R. Zhai, X. Xue, L. Zhang, X. Yang, L. Zhao, and C. Zhang, "Strain-specific anti-inflammatory properties of two Akkermansia muciniphila strains on chronic colitis in mice," Frontiers in Cellular and Infection Microbiology, vol. 9, p. 239, 2019.

[45] D. Srutkova, M. Schwarzer, T. Hudcovic et al., "Bifidobacterium longum CCM 7952 promotes epithelial barrier function and prevents acute DSS-induced colitis in strictly strain- 
specific manner," PLoS One, vol. 10, no. 7, article e0134050, 2015.

[46] Y. Zhao, C. Zhang, L. Yu et al., "Phylogenetic and comparative genomic analysis of Lactobacillus fermentum strains and the key genes related to their intestinal anti-inflammatory effects," Engineering, 2021.

[47] C. Dunne, L. O'Mahony, L. Murphy et al., "In vitro selection criteria for probiotic bacteria of human origin: correlation with in vivo findings," American Journal of Clinical Nutrition, vol. 73, no. 2, pp. 386S-392S, 2001.

[48] Y. Chen, Y. Jin, C. Stanton et al., "Dose-response efficacy and mechanisms of orally administered CLA-producing Bifidobacterium breve CCFM683 on DSS-induced colitis in mice," Journal of Functional Foods, vol. 75, no. 3, article 104245, 2020.

[49] M. Szulińska, I. Łoniewski, S. van Hemert, M. Sobieska, and P. Bogdański, "Dose-dependent effects of multispecies probiotic supplementation on the lipopolysaccharide (LPS) level and cardiometabolic profile in obese postmenopausal women: a 12 -week randomized clinical trial," Nutrients, vol. 10 , no. 6 , p. 773, 2018.

[50] X. W. Gao, M. Mubasher, C. Y. Fang, C. Reifer, and L. E. Miller, "Dose-response efficacy of a proprietary probiotic formula of Lactobacillus acidophilus CL1285 and Lactobacillus casei LBC80R for antibiotic-associated diarrhea and clostridium difficile -associated diarrhea prophylaxis in adult patients," American Journal of Gastroenterology, vol. 105, no. 7, pp. 1636-1641, 2010.

[51] R. Li, Y. F. Zhang, D. B. Polk, P. M. Tomasula, F. Yan, and L. S. Liu, "Preserving viability of Lactobacillus rhamnosus GG in vitro and in vivo by a new encapsulation system," Journal of Controlled Release, vol. 230, pp. 79-87, 2016.

[52] D. J. Silberger, C. L. Zindl, and C. T. Weaver, "Citrobacter rodentium: a model enteropathogen for understanding the interplay of innate and adaptive components of type $3 \mathrm{immu}-$ nity," Mucosal Immunology, vol. 10, no. 5, pp. 1108-1117, 2017.

[53] A. L. Glasser, J. Boudeau, N. Barnich, M. H. Perruchot, J. F. Colombel, and A. Darfeuille-Michaud, "Adherent invasive Escherichia coli strains from patients with Crohn's disease survive and replicate within macrophages without inducing host cell death," Infection and Immunity, vol. 69, no. 9, pp. 55295537, 2001.

[54] C. C. Chen, S. Louie, H. N. Shi, and W. A. Walker, "Preinoculation with the probiotic Lactobacillus acidophilus early in life effectively inhibits murine Citrobacter rodentium colitis," Pediatric Research, vol. 58, no. 6, pp. 1185-1191, 2005.

[55] R. N. Fedorak and K. L. Madsen, "Probiotics and the management of inflammatory bowel disease," Inflammatory Bowel Diseases, vol. 10, no. 3, pp. 286-299, 2004.

[56] C. Maciej, L. P. Hale, and L. Physiology, "Bacterial-mucosal interactions in inflammatory bowel disease-an alliance gone bad," American Journal of Physiology-Gastrointestinal and Liver Physiology, vol. 295, no. 6, pp. G1139-G1149, 2008.

[57] Y. Chen, Y. Jin, C. Stanton et al., "Alleviation effects of Bifidobacterium breve on DSS-induced colitis depends on intestinal tract barrier maintenance and gut microbiota modulation," European Journal of Nutrition, vol. 60, no. 1, pp. 369-387, 2021.

[58] G. Wang, Q. Xu, X. Jin et al., "Effects of Lactobacilli with different regulatory behaviours on tight junctions in mice with dextran sodium sulphate-induced colitis," Journal of Functional Foods, vol. 47, pp. 107-115, 2018.
[59] A. J. Leonel and J. I. Alvarez-Leite, "Butyrate: implications for intestinal function," Current Opinion in Clinical Nutrition and Metabolic Care, vol. 15, no. 5, pp. 474-479, 2012.

[60] H. Liu, J. Wang, T. He et al., "Butyrate: a double-edged sword for health?," Advances in Nutrition, vol. 9, no. 1, pp. 21-29, 2018.

[61] P. C. A. Moquet, L. Onrust, F. van Immerseel, R. Ducatelle, W. H. Hendriks, and R. P. Kwakkel, "Importance of release location on the mode of action of butyrate derivatives in the avian gastrointestinal tract," World's Poultry Science Journal, vol. 72, no. 1, pp. 61-80, 2016.

[62] T. Horiuchi, H. Mitoma, S.-I. Harashima, H. Tsukamoto, and T. Shimoda, "Transmembrane TNF- $\alpha$ : structure, function and interaction with anti-TNF agents," Rheumatology, vol. 49, no. 7, pp. 1215-1228, 2010.

[63] I. A. Lee, E. A. Bae, J. H. Lee et al., “Bifidobacterium longum HY8004 attenuates TNBS-induced colitis by inhibiting lipid peroxidation in mice," Inflammation Research, vol. 59, no. 5, pp. 359-368, 2010.

[64] A. K. Pandurangan, S. Ismail, Z. Saadatdoust, and N. M. Esa, "Allicin alleviates dextran sodium sulfate- (DSS-) induced ulcerative colitis in BALB/c mice," Oxidative Medicine \& Cellular Longevity, vol. 2015, article 605208, pp. 1-13, 2015.

[65] J. J. Hansen, L. Holt, and R. B. Sartor, "Gene expression patterns in experimental colitis in IL-10-deficient mice," Inflammatory Bowel Diseases, vol. 15, no. 6, pp. 890-899, 2009.

[66] S. K. Mittal and P. A. Roche, "Suppression of antigen presentation by IL-10," Current Opinion in Immunology, vol. 34, pp. 22-27, 2015.

[67] J. Yang, Q. Li, S. M. Henning et al., "Effects of prebiotic fiber xylooligosaccharide in adenine-induced nephropathy in mice," Molecular Nutrition \& Food Research, vol. 62, no. 15, article e1800014, 2018.

[68] N. Burger-van Paassen, A. Vincent, P. J. Puiman et al., "The regulation of intestinal mucin MUC2 expression by shortchain fatty acids: implications for epithelial protection," Biochemical Journal, vol. 420, no. 2, pp. 211-219, 2009.

[69] H. B. Wang, P. Y. Wang, X. Wang, Y. L. Wan, and Y. C. Liu, "Butyrate enhances intestinal epithelial barrier function via up-regulation of tight junction protein claudin-1 transcription," Digestive Diseases and Sciences, vol. 57, no. 12, pp. 3126-3135, 2012.

[70] L. Peng, Z. R. Li, R. S. Green, I. R. Holzman, and J. Lin, "Butyrate enhances the intestinal barrier by facilitating tight junction assembly via activation of AMP-activated protein kinase in Caco-2 cell monolayers," Journal of Nutrition, vol. 139, no. 9, pp. 1619-1625, 2009.

[71] X. Dou, N. Gao, D. Yan, and A. Shan, "Sodium butyrate alleviates mouse colitis by regulating gut microbiota dysbiosis," Animals, vol. 10, no. 7, article 1154, 2020.

[72] Y.-M. Lu, J.-J. Xie, C.-G. Peng, B.-H. Wang, K.-C. Wang, and L.-J. Li, "Enhancing clinical efficacy through the gut microbiota: a new field of traditional Chinese medicine," Engineering, vol. 5, no. 1, pp. 40-49, 2019.

[73] H. Chu, A. Khosravi, I. P. Kusumawardhani et al., "Genemicrobiota interactions contribute to the pathogenesis of inflammatory bowel disease," Science, vol. 352, no. 6289, pp. 1116-1120, 2016.

[74] H. L. Klein and K. N. Kreuzer, "Replication, recombination, and repair: going for the gold," Molecular Cell, vol. 9, no. 3, pp. 471-480, 2002. 
[75] J. Youell and K. Firman, "Mechanistic insight into type I restriction endonucleases," Frontiers in Bioscience, vol. 17, no. 7, pp. 2122-2139, 2012.

[76] J. M. Norman, S. A. Handley, M. T. Baldridge et al., "Diseasespecific alterations in the enteric virome in inflammatory bowel disease," Cell, vol. 160, no. 3, pp. 447-460, 2015.

[77] P. Liu, H. Zhang, Y. Wang et al., "Screening and characterization of an $\alpha$-L-fucosidase from Bacteroides fragilis NCTC9343 for synthesis of fucosyl-N-acetylglucosamine disaccharides," Applied Microbiology and Biotechnology, vol. 104, no. 18, pp. 7827-7840, 2020.

[78] J. E. Becerra, J. M. Coll-Marqués, J. Rodríguez-Díaz, V. Monedero, and M. J. Yebra, "Preparative scale purification of fucosyl-N-acetylglucosamine disaccharides and their evaluation as potential prebiotics and antiadhesins," Applied Microbiology and Biotechnology, vol. 99, no. 17, pp. 7165-7176, 2015.

[79] J. Spitz, R. Yuhan, A. Koutsouris, C. Blatt, J. Alverdy, and G. Hecht, "Enteropathogenic Escherichia coli adherence to intestinal epithelial monolayers diminishes barrier function," American Journal of Physiology-Gastrointestinal and Liver Physiology, vol. 268, no. 2, pp. G374-G379, 1995. 